\title{
Presencia internacional de los estudios sobre literatura catalana en el A\&HCI
}

\author{
Jordi Ardanuy*, LLuis Quintana**, Cristobal Urbano*
}

Resumen: Caracterización internacional desde el punto de vista bibliométrico y temático de los estudios sobre literatura catalana que resultan visibles a través del Arts \& Humanities Citation Index. El estudio se ha realizado fundamentalmente recuperando registros en esta base de datos a partir de una lista de autores literarios notables. Se constata la escasa cobertura que tienen los estudios de literatura catalana en dicha base de datos, lo que sugiere una escasa visibilidad internacional de la disciplina, así como un predominio de artículos en español. Además se confirman algunos resultados de trabajos anteriores sobre estudiosos (y su grado de coautoría) y sobre obras y épocas más estudiadas.

Palabras clave: bibliometria, humanidades, literatura catalana, A\&HCI, visibilidad internacional.

\section{International presence of studies on Catalan literature in the Arts \& Humanities Citation Index}

Abstract: This paper shows various thematic and bibliometric characteristics of studies on Catalan literature, visible internationally through the Arts E Humanities Citation Index. The study was performed primarily by retrieving records from this database, based on a list of major literary authors. The results reveal a low coverage of Catalan literature studies, suggesting poor international visibility for this discipline, as well as a preponderance of articles in Spanish. Furthermore some results from prior studies are confirmed, concerning type of authorship (and degree of co-authorship) and the most analyzed periods and works.

Keywords: bibliometrics, bumanities, Catalan literature, AEHCI.

\section{Introducción}

Arts \& Humanities Citation Index (A\&HCI) es una base de datos de citas de Thomson Scientific (antiguamente ISI), utilizada internacionalmente para obtener

* Departament de Biblioteconomia i Documentació. Universitat de Barcelona. Catalonia (Spain). Correo-e: jordi_ardanuy@ub.edu.

** Departament de Filologia Catalana. Universitat Autònoma de Barcelona. Catalonia (Spain). Correo-e: lluis.quintana@uab.es.

*:* Departament de Biblioteconomia i Documentació. Universitat de Barcelona. Catalonia (Spain). Correo-e: urbano@ub.edu.

Recibido: 15-6-09; aceptado: 19-11-09. 
referencias de artículos sobre arte y humanidades, que está en funcionamiento desde 1977 e integrada desde 2005 en el Web of Knowledge (WoK). Las bases de datos del Web of Knowledge ha sido tradicionalmente la principal plataforma para encontrar rápidamente información, analizarla y compartirla tanto en las ciencias, las ciencias sociales como las artes y humanidades y, aunque existen otras bases de datos susceptibles de ser analizadas como ISOC o DIALNET, éstas tienen una proyección más bien regional y Scopus una escasa cobertura en humanidades (Jacsó, 2009).

Sin embargo, pese a la vocación internacional de WoK, varios estudios han demostrado sus limitaciones en distintas áreas de las humanidades, debido a la importancia de las monografías en dichas áreas y al hecho de que la producción normalmente se hace en lenguas distintas al inglés (Luukkonen, 1991; Nederhof y Zwaan, 1991; Sanz y Martin, 1997; Moed, 2005; Nederhof, 2006; Fernández Izquierdo y otros, 2007; Ardanuy, 2008a). Estas limitaciones han ocasionado que los estudios documentales y bibliométricos de análisis de citas en humanidades sean escasos si se comparan con los de las ciencias experimentales (Ardanuy, 2009a).

Pese a las limitaciones comentadas, es posible mostrar ciertas características bibliométricas y temáticas de los estudios sobre literatura catalana y su nivel de visibilidad internacional a partir de una selección de registros en el A\&HCI, como mostramos en este artículo. En el presente trabajo nos referimos como literatura catalana únicamente a la realizada en catalán, independientemente del lugar de producción.

\section{Metodología}

El primer hecho que se constata al revisar las principales publicaciones especializadas en lengua y literatura catalanas y que constituyen el núcleo de la disciplina - esencialmente escritas en catalán-Els Marges, Caplletra, Estudis de Llengua i literatura catalanes, Estudis Romànics y Llengua E literatura es que ninguna de ellas está indexada en las bases de datos de Thomson Scientific (Ardanuy, 2008b). Descartada, pues, la posibilidad de encontrar artículos de estas fuentes, se procedió a buscar artículos sobre literatura catalana en el WoK publicados en otras revistas. La ecuación de búsquedas permite hacerlo por los siguientes campos: Topic (search topic terms within the fields Title, Abstract, Author y Keywords), Title, Author, Group Author, Publication Name, Year Published, Address, Language y Document Type. Además, de los operadores lógicos (AND, OR, NOT y SAME) y de los caracteres comodín (*, \$, ?) la búsqueda también puede limitarse por años.

Para recuperar los registros sobre literatura catalana se recurrió a una doble estrategia. Por una parte, se recuperaron documentos que incluyan en el campo Topic "Catalan Literature", "Catalan SAME Literature" o también "Catalan AND Literature.. Por otro lado, se realizó una búsqueda paralela a partir de una lista de autores literarios notables de la literatura catalana utilizados como tema (Topic). 
Una dificultad añadida en este último caso era la ausencia de un canon inapelable que incluyera a los autores de la literatura catalana más importantes. Desde un punto de vista meramente práctico se optó por seleccionar aquellos autores que superasen las 100 líneas de entrada en el Nou diccionari de la Literatura Catalana (NDLC) (Bou, 2000), de los que resultaron un total de 92 (Ardanuy, 2008a: 286). La cobertura cronológica del A\&HCI va desde 1975 hasta la actualidad, aunque con una actualización de las novedades variable. En consecuencia, se explotaron todos los registros entre 1975 y 2007. Posteriormente se eliminaron los registros no pertinentes.

Es necesario mencionar algunos problemas. Es el caso de las variantes de nombres de autores literarios clásicos, como ocurre con Ramon Llull (Raymond Llull, Raymond Lully, Raimundus Lulius...). También, la dificultad de distinguir en algunos casos los estudios sobre autores literarios que tienen un mismo apellido, por lo que tuvo que realizarse un filtrado posterior en el que siempre hay cabida para errores.

Otro aspecto a considerar es el del control de autoridades, que no es estricto en el A\&HCI, de manera que los autores de los trabajos pueden aparecer de maneras diferentes. Para resolver estas incertidumbres se recurrió a bases de datos alternativas, cuando fue menester.

Los registros seleccionados del A\&HCI se exportaron a un gestor de referencias para su control de autoridades y, posteriormente, a un gestor de bases de datos relacional para un tratamiento estadístico posterior.

\section{Resultados y discusión}

El número de registros obtenido después del proceso de filtrado fue de 679, un número pequeño si se considera que se han explorado un total de 92 autores literarios, además del tema general (topic) "Catalan Literature" en alguna de las variantes comentadas supra, de lo que se puede colegir una visibilidad internacional muy limitada si se compara, por ejemplo, con los 248 registros que se obtienen solamente para Antonio Machado, para el mismo período.

De los 679 registros obtenidos, solamente 79 (11,6\%) se obtuvieron directamente a través del tópico "Catalan Literature" en alguna de las variantes comentadas. Una buena parte de estos registros también se recuperaron a partir de la lista de autores, aunque por supuesto solamente fueron contabilizados una vez. Este porcentaje escaso 11,6\%, además de unos guarismos mucho más exiguos obtenidos en las pruebas con otras variantes como "Catalan Novel" O "Catalan Poetry" y el solapamiento de registros con los ya obtenidos nos llevaron a desistir de seguir probando con todos los géneros literarios posibles.

Si se analiza el tipo de documento al que hacen referencia los registros encontrados, la mayor parte (67\%) pertenecen a reseñas de libros, mientras que solamente un $28,9 \%$ se clasifican como artículos (tabla I). El porcentaje restante se diversifica en notas, aspectos biográficos, reseñas teatrales y material editorial, 
que engloba elementos documentales heterogéneos, como pueden ser entrevistas u opiniones.

TABLA I

Distribución de registros según el tipo de documentos

\begin{tabular}{l|c|c}
\multicolumn{1}{c|}{ Tipo de documento } & $\begin{array}{c}\mathbf{N}^{\mathbf{0}} \text { de } \\
\text { registros }\end{array}$ & $\begin{array}{c}\text { \% de } \\
\text { registros }\end{array}$ \\
\hline Reseña de libro (Book Review) & 455 & 67,0 \\
\hline Artículo (Article) & 196 & 28,9 \\
\hline Elementos sobre un individuo (Item About an Individual) & 7 & 1,0 \\
\hline Material editorial (Editorial material) & 7 & 1,0 \\
\hline Elementos biográficos (Biographical-Item) & 6 & 0,9 \\
\hline Nota (Note) & 5 & 0,7 \\
\hline Reseña de teatro (Theater Review) & 3 & 0,4 \\
\hline Total & 679 & 100 \\
\hline
\end{tabular}

La figura 1 recoge la evolución cronológica de los libros y artículos cuyas referencias se localizan en el A\&HCI según su año de publicación. Los datos se han agrupado en tres períodos. Se observa un incremento ligero, pero permanente, del número de artículos, mientras que las reseñas de libros decrecen claramente en el período 1997-2007. El gráfico muestra también el total de registros,

FIGURA 1

Evolución cronológica del tipo de documento en los registros del AEHCI

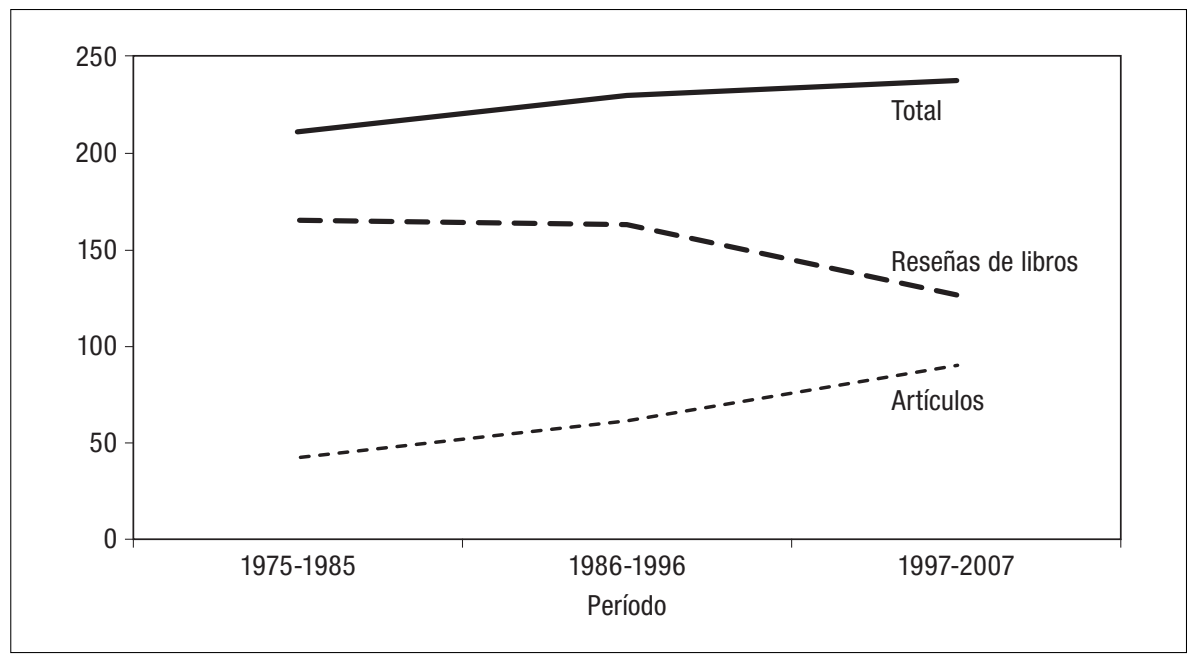


con un incremento atenuado en el último período debido a la disminución ya comentada de reseñas.

Este incremento tan pequeño en el número de artículos muestra que el estudio de la literatura catalana no está ganando protagonismo internacional.

Con respecto a la lengua en la que están publicados, el inglés es la dominante con un 54,5\% de referencias (tabla II), seguida del español (28,4\%), mientras que al catalán solamente le corresponden un 2,1\% de los registros, hecho que se explica de manera sencilla si se considera, como se ha comentado anteriormente, la ausencia en el A\&HCI de revistas específicas de lengua y literatura o de humanidades en catalán. Las escasas referencias en catalán aparecen en publicaciones generales sobre temas hispánicos, o más específicas, de literatura hispánica o bien románica que han admitido colaboraciones en varias lenguas, como en el caso del Bulletin of Hispanic Studies. De todas maneras, esto demuestra que, aunque no se indexen las revistas catalanas de literatura, no es del todo imposible publicar trabajos sobre literatura catalana en catalán y aparecer en el A\&HCI.

TABLA II

Distribución de lenguas según el tipo de documento

\begin{tabular}{l|c|c|c|c|c|c|c|c}
\hline \multirow{2}{*}{ Lengua } & \multicolumn{2}{|c|}{ Total } & \multicolumn{2}{c|}{ Artículo } & Reseña de libro & \multicolumn{2}{c}{ Otros } \\
\cline { 2 - 10 } & No & $\mathbf{0}$ & No & $\mathbf{0}$ & No & $\mathbf{\%}$ & No & \% \\
\hline Inglés & 370 & 54,5 & 68 & 34,7 & 297 & 65,3 & 5 & 17,9 \\
\hline Español & 193 & 28,4 & 104 & 53,1 & 72 & 15,8 & 17 & 60,7 \\
\hline Francés & 53 & 7,8 & 11 & 5,6 & 38 & 8,4 & 4 & 14,3 \\
\hline Alemán & 35 & 5,2 & 4 & 2,0 & 30 & 6,6 & 1 & 3,6 \\
\hline Catalán & 14 & 2,1 & 4 & 2,0 & 9 & 2,0 & 1 & 3,6 \\
\hline Italiano & 14 & 2,1 & 5 & 2,6 & 9 & 2,0 & 0 & 0,0 \\
\hline Total & 679 & 100 & 196 & 100 & 455 & 100 & 28 & 100 \\
\hline
\end{tabular}

Ahora bien, como muestra la figura 2, el peso de las lenguas ha ido variando con el tiempo, de manera que las aportaciones en catalán han aumentado tímidamente, mientras que el español ha ido ganando terreno al inglés.

Respecto a las publicaciones con más registros (tabla III), en primer lugar se encuentra World Literature Today, con un total de 18,3\%, seguida a gran distancia por el Bulletin of Hispanic Studies (8,7\%) y Ínsula. Revista de Letras y Ciencias Humanas $(7,4 \%)$.

Sin embargo la importancia de World Literature Today se debe exclusivamente a las reseñas de libros realizadas para dar a conocer a la comunidad anglófona e internacional novedades editoriales sobre literatura catalana. Excluidas estas y todos los otros materiales que no sean específicamente artículos, el mayor por- 
FIGURA 2

Evolución cronológica del porcentaje de registros según la lengua del original

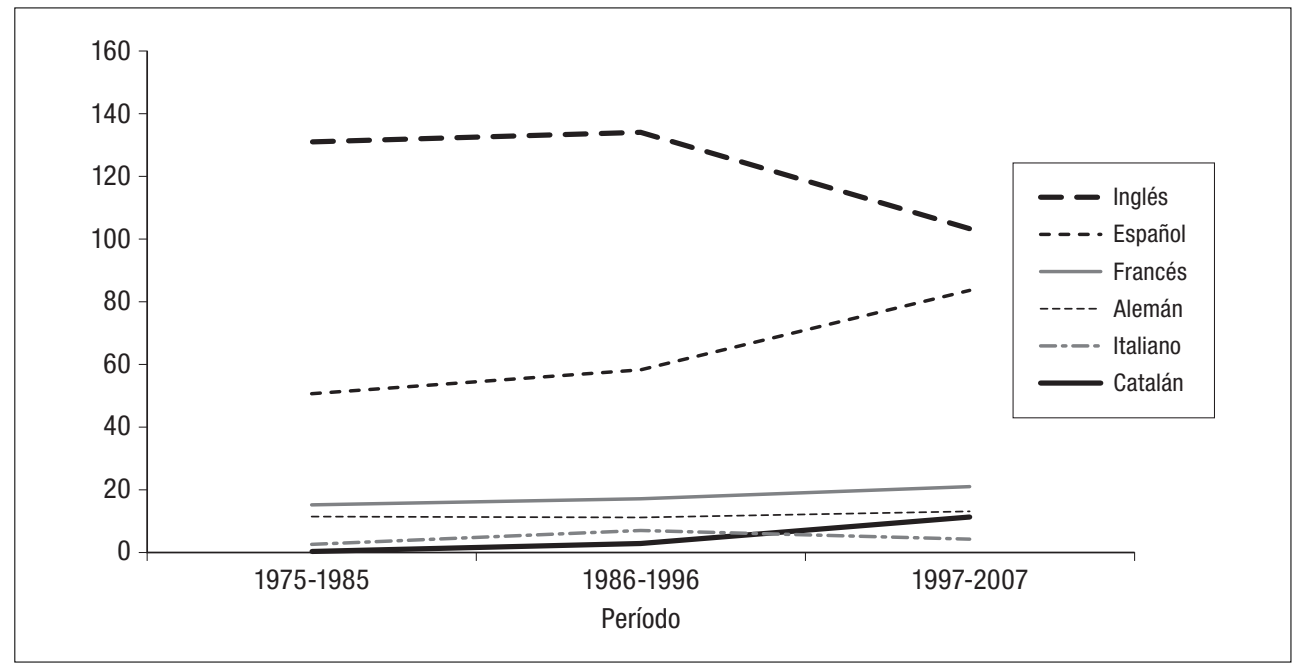

TABLA III

Publicaciones con más registros

\begin{tabular}{|c|c|c|c|c|}
\hline Publicación & $\begin{array}{c}\mathrm{N}^{\mathbf{o}} \\
\text { de } \\
\text { registros }\end{array}$ & $\begin{array}{c}\% \\
\text { de } \\
\text { registros }\end{array}$ & $\begin{array}{c}\text { Lugar } \\
\text { de } \\
\text { publicación }\end{array}$ & $\begin{array}{c}\text { Lenguas que declaran } \\
\text { admitir en sus } \\
\text { contribuciones actualmente }\end{array}$ \\
\hline World Literature Today & 124 & 18,3 & EE.UU & Inglés. \\
\hline Bulletin of Hispanic Studies & 59 & 8,7 & Reino Unido & $\begin{array}{l}\text { Inglés y español. Pero hay } \\
\text { textos en catalán. }\end{array}$ \\
\hline $\begin{array}{l}\text { Insula. Revista de Letras y Cien- } \\
\quad \text { cias Humanas }\end{array}$ & 50 & 7,4 & España & Español. \\
\hline Cuadernos Hispanoamericanos & 42 & 6,2 & España & Español. \\
\hline Modern Language Review & 27 & 4,0 & Reino Unido & Inglés. \\
\hline Hispanic Review & 24 & 3,5 & EE.UU & Inglés y lenguas románicas. \\
\hline $\begin{array}{l}\text { Anales de la Literatura española } \\
\text { contemporánea }\end{array}$ & 22 & 3,2 & EE.UU & Inglés y español. \\
\hline $\begin{array}{l}\text { Hispania. A Journal devoted to } \\
\text { the teaching of Spanish and } \\
\text { Portuguese }\end{array}$ & 15 & 2,2 & EE.UU. & Español, portugués e inglés. \\
\hline $\begin{array}{l}\text { Zeitschrift für Romanische Pbi- } \\
\quad \text { lologie }\end{array}$ & 15 & 2,2 & Alemania & $\begin{array}{l}\text { Alemán, francés, italiano, } \\
\text { español y portugués. }\end{array}$ \\
\hline Otras 106 & 301 & 44,3 & - & - \\
\hline Total & 679 & 100 & 一 & 一 \\
\hline
\end{tabular}


centaje de referencias recae en Ínsula. Revista de Letras y Ciencias Humanas y en Cuadernos Hispanoamericanos (tabla IV). Ninguna de estas revistas es relevante para el núcleo de la disciplina según se desprende de un estudio anterior basado en un análisis de citaciones (Ardanuy y otros, 2008b).

Así, como denota la ya citada tabla II, si solamente se consideran los artículos, predominan las publicaciones en español por encima del inglés.

\section{TABLA IV}

Publicaciones con más artículos

\begin{tabular}{|c|c|c|c|c|}
\hline Publicación & $\begin{array}{c}\mathbf{N}^{\mathbf{o}} \\
\text { de } \\
\text { artículos }\end{array}$ & $\begin{array}{c}\% \\
\text { de } \\
\text { artículos }\end{array}$ & $\begin{array}{l}\text { Lugar } \\
\text { de } \\
\text { publicación }\end{array}$ & $\begin{array}{l}\text { Lenguas que declaran } \\
\text { admitir en sus } \\
\text { contribuciones actualmente }\end{array}$ \\
\hline $\begin{array}{l}\text { Ínsula. Revista de Letras y Cien- } \\
\quad \text { cias Humanas }\end{array}$ & 28 & 14,3 & España & Español. \\
\hline Cuadernos Hispanoamericanos & 23 & 11,7 & España & Español. \\
\hline $\begin{array}{l}\text { Anales de la Literatura Española } \\
\text { Contemporánea }\end{array}$ & 11 & 5,6 & EE.UU. & Inglés y español. \\
\hline Bulletin of Hispanic Studies & 11 & 5,6 & Reino Unido & Inglés y español. \\
\hline Revista de Occidente & 11 & 5,6 & España & Español \\
\hline Romance Quarterly & 9 & 4,6 & EE.UU. & Inglés y lenguas románicas. \\
\hline Hispanofila & 8 & 4,1 & EE.UU. & Inglés, español y portugués. \\
\hline Modern Language Review & 7 & 3,6 & Reino Unido & Inglés. \\
\hline Crítica Hispánica & 6 & 3,1 & EE.UU. & $\begin{array}{l}\text { Inglés, español. Pero hay } \\
\text { textos en catalán. }\end{array}$ \\
\hline Romance Notes & 5 & 2,6 & EE.UU. & Inglés y lenguas románicas. \\
\hline Otras 55 & 77 & 39,3 & - & - \\
\hline Total & 196 & 100 & - & - \\
\hline
\end{tabular}

Respecto a los autores de las publicaciones, en el caso de las reseñas de libros destaca notablemente con 54 aportaciones (11,9\%) Albert M. Forcadas, del Departamento de Lenguas modernas i Estudios culturales de la University of Alberta. Igual que en su caso, la mayor parte de reseñas realizadas en inglés corresponden a investigadores vinculados a universidades fuera de la catalanofonía.

$\mathrm{Si}$ nos referimos a los siete principales productores de artículos (tabla V), a excepción de Albert Mament, ninguno resulta relevante respecto al número de citas que recibe en los estudios bibliométricos sobre la literatura catalana (Ardanuy y otros, 2008b). Todos estos seis han alternado sus estudios de literatura catalana con otros de literatura y cultura hispana, en una mayor o menor intensidad. El caso de Manent es diferente, y sus trabajos indexados, sobre la obra de Josep Carner, fueron todos publicados en el año 1984. 
TABLA V

Autores con más artículos

\begin{tabular}{l|c|l}
\hline \multicolumn{1}{c|}{ Autor } & $\begin{array}{c}\mathbf{N}^{\mathbf{0}} \text { de } \\
\text { artículos }\end{array}$ & \multicolumn{1}{|c}{ Adscripción geográfica } \\
\hline David J. Viera & 4 & EE.UU. \\
\hline D. Gareth Walters & 4 & Reino Unido \\
\hline Dominic Keown & 3 & Reino Unido \\
\hline Geraldine C. Nichols & 3 & EE.UU \\
\hline Josefina González & 3 & EE.UU \\
\hline Jaume Pont Ibánez & 3 & Cataluña (España) \\
\hline Albert Manent Segimon & 3 & Cataluña (España) \\
\hline
\end{tabular}

Indicamos también que solamente se han encontrado dos artículos realizados en colaboración entre dos autores. Este nivel escaso de coautoría suele ser habitual en las disciplinas de humanidades (Wiberley; 1994; Bourke 1997; Molteni y Zulueta, 2002; Lowe, 2003) y ya se había observado en el caso de la literatura catalana (Ardanuy, 2008a).

Si se observa la distribución de artículos en las grandes áreas en que se divide el estudio de la literatura catalana y su historia (Molas, 2001), observamos (tabla VI) un predominio global abrumador de la literatura contemporánea (80,2\%). De estos, el 60,4\% son en español y un 30,6\% en inglés. Una presencia tan escasa de artículos en inglés dificulta a los especialistas de otras literaturas, especialmente no románicas, que puedan tener un conocimiento detallado de la producción contemporánea catalana. Este predominio, aunque más atenuado, también se ha detectado en estudios realizados sobre análisis de citas y de tesis doctorales (Ardanuy y otros, 2008b; Ardanuy y otros, 2009b).

\section{TABLA VI}

Distribución de artículos en les grandes áreas de estudio de la literatura catalana y su bistoria

\begin{tabular}{|c|c|c|c|c|c|c|c|c|}
\hline \multirow{2}{*}{$\begin{array}{l}\text { Períodos } \\
\text { históricos }\end{array}$} & \multirow{2}{*}{$\begin{array}{c}\mathrm{N}^{\mathbf{0}} \\
\text { de } \\
\text { artículos }\end{array}$} & \multirow{2}{*}{$\begin{array}{c}\% \\
\text { de } \\
\text { artículos }\end{array}$} & \multicolumn{6}{|c|}{$\mathrm{N}^{\circ}$ de artículos según la lengua } \\
\hline & & & Catalán & Inglés & Francés & Alemán & Italiano & Español \\
\hline Contemporánea & 134 & 80,2 & 3 & 41 & 5 & 2 & 2 & 81 \\
\hline Medieval & 33 & 19,8 & 1 & 17 & 4 & 2 & 3 & 6 \\
\hline Moderna & 0 & 0 & 0 & 0 & 0 & 0 & 0 & 0 \\
\hline
\end{tabular}

En el caso de la literatura medieval, aunque es menos significativa globalmente $(19,8 \%)$, se observa un predominio de los escritos en inglés (51,3\%). Indiquemos también que no hemos encontrado ningún artículo relacionado con la lite- 


\section{TABLA VII}

Obras literarias estudiadas con más artículos específicos

\begin{tabular}{l|c|c|c|c}
\hline \multicolumn{1}{c|}{ Obras literarias } & $\begin{array}{c}\mathbf{N}^{\mathbf{o}} \text { total } \\
\text { de artículos }\end{array}$ & $\begin{array}{c}\mathbf{N}^{\circ} \text { de artículos } \\
\text { en inglés }\end{array}$ & $\begin{array}{c}\mathbf{N}^{\circ} \text { de artículos } \\
\text { en español }\end{array}$ & $\begin{array}{c}\mathbf{N}^{\circ} \text { de artículos } \\
\text { en alemán }\end{array}$ \\
\hline Tirant lo Blanc & 7 & 3 & 2 & 2 \\
\hline La plaça del Diamant & 6 & 5 & 1 & 0 \\
\hline El carrer de les Camèlies & 2 & 0 & 2 & 0 \\
\hline La Bogeria & 2 & 0 & 2 & 0 \\
\hline Ramona, adéu & 2 & 1 & 1 & 0 \\
\hline
\end{tabular}

ratura de la edad moderna aunque hay que considerar que ninguno de los autores utilizados en nuestra lista pertenece a esa época por el simple hecho de que los autores de esta época raramente se consideran canónicos (de hecho, la historiografia literaria solía llamar a los siglos XVI-XVIII "época de decadencia").

La tabla VII ofrece las obras literarias a las que se han dedicado más artículos específicos. En primer lugar figura Tirant lo Blanc, coincidiendo con otros estudios que la sitúan como la obra literaria más estudiada de la literatura catalana (Ardanuy y otros, 2008b; Ardanuy y otros, 2009b).

Entre los autores literarios (tabla VIII) destaca en primer lugar Mercè Rodoreda, seguida de Salvador Espriu y Montserrat Roig. Este ranking no coincide

\section{TABLA VIII}

Autores literarios sobre los que se ban publicado más artículos

\begin{tabular}{|c|c|c|c|c|c|}
\hline Autores literarios & $\begin{array}{c}\mathrm{N}^{\mathbf{2}} \\
\text { total } \\
\text { de artículos }\end{array}$ & $\begin{array}{c}\mathrm{N}^{\mathbf{2}} \\
\text { de artículos } \\
\text { en inglés }\end{array}$ & $\begin{array}{c}\mathrm{N}^{\mathbf{2}} \\
\text { de artículos } \\
\text { en español }\end{array}$ & $\begin{array}{c}\mathrm{N}^{\mathrm{o}} \\
\text { de artículos } \\
\text { en catalán }\end{array}$ & $\begin{array}{c}\mathrm{N}^{\circ} \text { de artículos } \\
\text { en italiano, } \\
\text { francés } \\
\text { y alemán }\end{array}$ \\
\hline Mercè Rodoreda & 17 & 10 & 7 & 0 & 0 \\
\hline Salvador Espriu & 10 & 4 & 5 & 0 & 1 \\
\hline Montserrat Roig & 8 & 6 & 2 & 0 & 0 \\
\hline Gabriel Ferrater & 7 & 1 & 5 & 0 & 1 \\
\hline Joanot Martorell & 7 & 3 & 2 & 0 & 2 \\
\hline Francesc Eiximenis & 6 & 4 & 1 & 0 & 1 \\
\hline Josep Carner & 6 & 0 & 6 & 0 & 0 \\
\hline Josep Vicent Foix & 6 & 2 & 3 & 0 & 1 \\
\hline Joan Brossa & 5 & 1 & 3 & 1 & 0 \\
\hline Joan Maragall & 5 & 1 & 2 & 1 & 1 \\
\hline Llorenç Villalonga & 5 & 2 & 3 & 0 & 0 \\
\hline Narcís Oller & 5 & 1 & 4 & 0 & 0 \\
\hline
\end{tabular}


cualitativamente con el de otros estudios ya mencionados (Ardanuy y otros, 2008b; Ardanuy y otros, 2009b), centrados en la investigación en los territorios catalanófonos.

\section{Conclusiones}

El presente trabajo ha constatado la escasa cobertura que tienen los estudios de literatura catalana en el A\&HCI lo que dificulta un estudio bibliométrico basado en el análisis de citas. Este hecho se justifica porque ninguna de las publicaciones especializadas en lengua y literatura catalanas que constituyen el núcleo de la disciplina está indexada en las bases de datos de Thomson Scientific, como ya se había puesto de relieve (Ardanuy, 2008b). Un número tan pequeño de registros sugiere un pobre escaso nivel de visibilidad de la disciplina a nivel internacional.

Pese a no disponer de un volumen abundante de registros, su análisis aporta cierta información sobre la disciplina y permite comparaciones con los estudios a los que complementa.

Los resultados obtenidos muestran un predominio de las reseñas de libros escritas en inglés por especialistas en filología catalana que trabajan especialmente en países anglófonos y que dan a conocer novedades bibliográficas publicadas en los territorios de habla catalana.

En cuanto a los artículos, publicados principalmente en español, se constata un predominio de los estudios de literatura contemporánea, lo que coincide con lo observado en trabajos anteriores sobre la investigación en literatura catalana en los territorios catalanófonos. Igualmente ocurre con la obra literaria más analizada, Tirant lo Blanc.

Pero también se aprecian diferencias. Así los investigadores más citados en las revistas de literatura catalana adolecen de cualquier registro en el A\&HCI, mientras que los que aquí aparecen, no ocupan lugares tan relevantes. Este hecho pone en evidencia que los trabajos más importantes de la literatura catalana carecen de la necesaria internacionalización y no se dan a conocer a los estudiosos de otras literaturas o de las humanidades en general.

Entre los autores literarios más estudiados destacan Mercè Rodoreda y Montserrat Roig, con un peso relativo considerablemente mayor que el observado en los estudios de citas y de tesis doctorales en los Países Catalanes. Este interés parece que se explica en parte por los estudios de literatura y género, menos abordados en la catalanofonía.

Finalmente indiquemos que la presencia de artículos de literatura catalana en cierto número de revistas indexadas en el A\&HCI debe ser una ventana que aprovechen los autores que estudian este ámbito del conocimiento, como estrategia complementaría a la difusión de sus trabajos en revistas específicas del área. El hecho de que las revistas de literatura catalana no estén indexadas a A\&HCI, no ha impedido una presencia de referencias que, aunque pequeña, podría ser 
más elevada si se tuviera una estrategia más clara de internacionalización de la investigación en este dominio. El trabajo demuestra en este sentido, por ejemplo, un déficit en el ámbito de la literatura moderna, que podría justificar una acción más decidida en las políticas dirigidas a los investigadores de literatura catalana de cara a una mejor orientación de la difusión de sus investigaciones. La lista de revistas obtenidas en este estudio, sin duda puede ser un punto de referencia para estos autores.

\section{Bibliografía}

Ardanuy, Jl. (2008a): Anàlisi bibliomètrica de la producció científica en literatura catalana [en línea]. Barcelona; Universitat de Barcelona, 2008 [consultado el 30 de octubre de 2009]. Disponible en http://www.tesisenred.net/TDX/TDX_UB/TESIS/AVAILABLE/ TDX-0226108-124023.

Ardanuy, J.; Quintana, L., y Urbano, C. (2008b): Una aproximació bibliomètrica a la recerca en literatura catalana. Randa, $\mathrm{n}^{\circ} 86,41-66$.

Ardanuy, J. (2009a): Els estudis bibliomètrics basats en l'anàlisi de citacions en àrees d'humanitats en el període 1959-2008 [en línea]. Bid: textos universitaris de biblioteconomia i documentació, $\mathrm{n}^{\circ} 22$ [consultado el 30 de octubre de 2009]. Disponible en http://www.ub.edu/bid.

Ardanuy, J.; Urbano, C., y Quintana, L. (2009b): The evolution of recent research on Catalan literature through the production of $\mathrm{PhD}$ theses: a bibliometric and social network análisis [en línea] Information Research, vol. 14 (2) [Consultado el 30 de octubre de 2009]. Disponible en http://informationr.net/ir/14-2/infres142.html.

Bou, E. (dir.) (2000): Nou diccionari de la Literatura Catalana. Barcelona: Edicions 62.

Bourke, P. (1997): Discipline Boundaries in the Social Sciences. Canberra: Academy of the Social Sciences.

Fernández Izquierdo, F.; Román, A.; Rubio, M. C.; Moreno, F. J.; Martín, C.; García, J. C.; Lascurain, M. L.; García, P. E.; Povedano, E., y Sanz, E. (2007): Bibliometric study of early Modern History in Spain based on bibliographic references in national scientific journals and conference proceedings. 11 International Conference of the International Society for Scientometrics and Informetrics, vol. 1, 266-271. Madrid: CSIC.

Jacsó, P. (2009): Scopus. Péter's Digital Reference shelf [en línea]. En Péter's Digital Reference Shelf [consultado el 30 de octubre de 2009]. Disponible en www.gale.cengage. com/reference/peter/200906/scopus.html.

Lowe, M. S. (2003): Reference analysis of the "American Historical Review". Collection Building, vol. 22 (1), 13-20.

Luukkonen, T. (1991): Soveltuuko viiteanalyysi tutkimuksen arviointiin?', Sosiaalilääketieteellinen Aikakauslehti, no 28, 189-196.

Moed, H. F. Ed. (2005): Citation Analysis in Research Evaluation Dordrecht: Springer.

Molas, J. (2001): Reports de la recerca a Catalunya: literatura catalana [en línea]. Barcelona: Institut d'estudis Catalans [consultado el 30 de octubre de 2009]. Disponible en http://www.iec.cat/gc/digitalAssets/500_3680_1097660898265_filologia.pdf. 
Molteni, V., y Zulueta, M. A. (2002): Análisis de la visibilidad internacional de la producción científica argentina en las Bases de datos Social Science Citation Index y Arts and Humanities Citation Index de 1990-2000. Revista Española de Documentación Cientifica, vol. 25 (4), 455: 465.

Nederhof, A. J., y Zwaan, R. A. (1991): Quality judgments of journals as indicators of research performance in the humanities and the social and behavioral sciences. Journal of the American Society for Information Science and Technology, vol. 42 (5), 332-340.

Nederhof, A. J. (2006): Bibliometric monitoring of research performance in the Social Sciences and the Humanities: a review. Scientometrics, vol. 66 (1), 81-100.

Quick, A. (2009): SCOPUS no longer available - but use Web of Knowledge [en línea]. En Library news and updates from the University of Abertay Dundee [consultado el 30 de octubre de 2009]. Disponible en http://libertay.blogspot.com/2008/06/scopus-no-longer-available-but-use-web.html.

Sanz, E., y Martín, C. (1997): Técnicas bibliométricas aplicadas a los estudios de usuarios. Revista general de información y documentación, vol. 7 (2), 41-68.

Wiberley, S. E., y Jones, W. G. (1994): Humanists revisited: a longitudinal look at the adoption of information technology. College and Research Libraries, vol. 55 (6), 499-509. 